\title{
Preparation for the accreditation according to ISO 15 189:A quality folder realization
}

\begin{abstract}
The quality manual is a quality guideline which is imposed to all medical laboratories which are ISO 15189 certified. In order to meet the standard requirements, we have elaborated a quality manual. This approach will enable the mastery of all LABCAPM (laboratory of toxicology and Pharmacology of the Center of Poison Control and Pharmacovigilance of Morocco) specific activities and as well optimize their realization. This article describes the methodology, the means used and the advantages of elaborating a quality manual in the context of an ISO 15189 accreditation procedure. In order to ensure this, we have developed several points in this article, starting with the presentation of the ISO 15189 standard, its requirements and topics, in the second chapter we described the standards used in the accreditation of ISO 15189. The quality manual: imposed by ISO 15189 "describe the content of a quality manual according to the ISO15189 standard. In results and discussion we presented the quality manual that we realized within the LAB-CAPM.
\end{abstract}

Volume 2 Issue 2 - 2018

\author{
Marouane Nasser,' Tijane M'hamed,' Narjis \\ Badrane, ${ }^{2}$ Soulaymani Rachida Bencheikh, ${ }^{2} \mathrm{El}$ \\ Bouazzi Omaima ${ }^{2}$ \\ 'Laboratory of biochemistry and immunology of Faculty of \\ Sciences, University Mohammed-V, Morocco \\ ${ }^{2}$ Laboratory of toxicology and pharmacology of the Center of \\ Poison Control and Pharmacovigilance of Morocco, Morocco
}

Correspondence: Nasser Marouane, Laboratory of biochemistry and immunology of Faculty of Sciences, University Mohammed-V, Morocco, Email marouane.nasser@wanadoo.fr

Received: March 16, 2018 | Published: April 24, 2018

Keywords: quality manual, ISO 15189, accreditation, quality assurance, medical laboratories

\section{Introduction}

Accreditation is a formal recognition of the competence of a laboratory, an inspection body or a certifying body to carry out specific activities which are well defined and which are examined throughout the accreditation audit. It's, in a way, a second level control. This competence recognition is intented to guarantee to the accredited bodies clients, that the deliveries of these accredited bodies, under cover their accreditation, are trustworthy. ${ }^{1}$ Accreditation might be as a part of a voluntary initiative, today it's the case for LAB-CAPM that's committed to obtain ISO 15189 accreditation.

Traceability is the key to quality. If their actions and reactions become quality related, then expensive failures and the accumulation of hidden costs may be reduced to an acceptable minimum or even prevented altogether. Quality folder is a holistic concept, which requires quality motivation of all people in an organization towards a common quality goal.

\section{Materials and methods}

We used ISO 15189 as materiel. The methods followed in the drafting of our quality manual are as follows:

a. Preparing laboratory for accreditation with the ISO 15189 standard;

b. Following chapter 4.2.2 of ISO 15189 which describes the structure of the manual documentation;

c. Writing the processes used in the laboratory.

\section{NF EN ISO I 5 I 89 standard}

It's a more specific standard as its title indicates: «Medical laboratories - Requirements for quality and competence».

This standard combines EN ISO 9001: 2000 standard quality system requirements and the technical requirements proper to medical laboratories. Part on «Management requirements» and a parton «Technical requirements» which takes into account the overall analysis including both pre-analytical and post-analytical phases; its summary is close to NF EN ISO/CEI 17025 standard.

The topics addressed on the part «Management requirements» are already mentioned in NF EN ISO 9001 standard (preventive actions, corrective actions, management review, audits, etc.). In contrast, chapters «Examination by referral laboratories», «External services and supplies» and «Advisory services» are specific. Technical requirements concern pre-analytical, analytical and postanalytical procedures, and as well results reporting, staff, facilities, environmental conditions and laboratory equipments. This is the first specific standard that covers the entire activities of medical laboratories. ${ }^{2}$ Laboratory accreditation use criteria and procedures specifically developed to determine technical competence.

Specialist technical assessors conduct a thorough evaluation of all factors in a laboratory that affect the production of test or calibration data. The criteria are based on the international standards called ISO/ IEC 17025 or ISO 15189, which are used for evaluating laboratories throughout the world. Laboratory accreditation bodies use this standard specifically to assess factors relevant to the laboratory's technical competence. ${ }^{3}$

\section{Preparation for accreditation}

Medical laboratory quality systems can be based on different standards or protocols, such as ISO 15189, ISO 17025, ISO9001, JCI, EFQM Model, and WHO Health Promoting Hospitals. The laboratory has to decide to which system it should apply. ${ }^{4}$ The most recent, internationally accepted Accreditation System is the ISO 15189 Standard. ${ }^{5}$ Previously, medical laboratories were accredited according to ISO/EN 45001, ISO/IEC 17025 standards. Top management of the candidate laboratory has to be committed to accreditation and fully support the team in every aspect. Large spectrum servicing 
laboratories have to establish an accreditation team. Coordination of these activities may be too large and detailed task for one manager. One person should be devoted and responsible for the development and maintenance of the Quality System Documents. Candidate laboratory has to obtain an official copy of ISO15189: 2007. Content of the standard should be understood very well. Criteria should be discussed by the maximum possible number of related staff. Weak and strong points of the existing practice should be identified. Weaknesses must be revised. Organizational arrangements, practices and procedures have to be examined to see whether they meet the requirements of the standard. Improvements have to be made on the basis of evaluation, if necessary. This stage is the most critical issue of preparation for accreditation. You have to evaluate your system. Decide on the correction. Document it and follow it up in a sufficient period of time. Then, you can decide whether your laboratory is ready for accreditation application or not. It may take more time, effort and money than you expect, to adopt the existing practice to the requirements of the standard.

\section{The quality manual: imposed by ISO I 5 I 89}

The quality manual is on the documents required by ISO 15189 . It's defined by ISO 9000 as a document which specifies the quality management system of an organization. The quality manual is a «Framework document» of the documentary structure. This documentary structure is often presented in the form of a pyramid (see below) where in the top of the pyramid there's the quality manual and in its base there's the records. Documents types, their designation, and as well the quality manual form are left up to every organization so it elaborates its own adapted document structure. Therefore, a small size organization might find it appropriate to include a complete description of its quality system in the quality manual, including all the documented procedures required by the standard. In the opposite, big size organizations might need a more important number of documentary levels, for example national procedures, local procedures, sectorial methods of operation, and perhaps even many quality manuals (group quality manual, site quality manual...) (Figure $1)$.

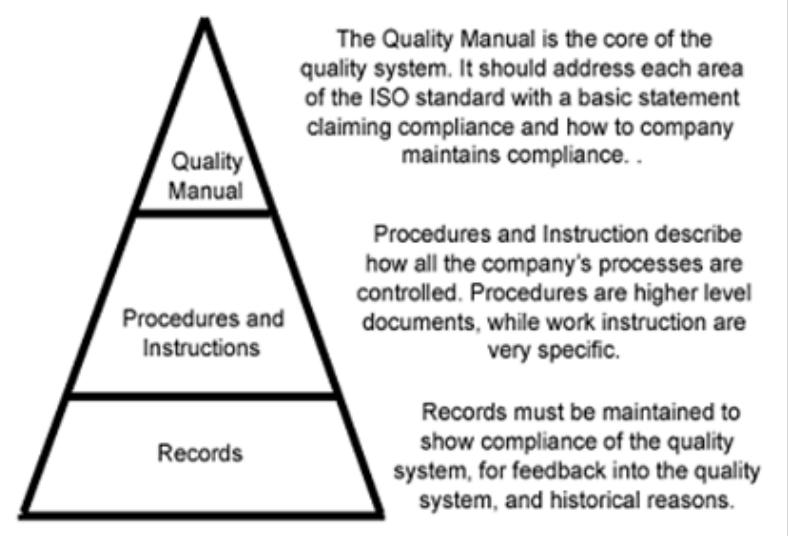

Figure I The ISO I5I89 documentation pyramid.

According to chapter 4.2.2 of the ISO 15189 standard, a quality manual should describe the quality management system and its documentation structure. The quality manual should include or make reference to supporting procedures; including technical procedures. It should highlight the documentation structure of the quality management system. The role and the responsibilities of the technical direction and the quality manager, including their responsibilities concerning the compliance to this international standard, should be included to the quality manual as well. The whole staff should be trained to the use and application of the quality manual, the referenced documents and all the relating requirements for their implementation. The quality manual should be kept up-to-date under the authority and the responsibility of a quality manager designated by the laboratory direction.

The table of contents of a medical laboratory quality manual might, for example, be presented as follow:

a) Introduction.

b) Laboratory description, its legal status, resources and main activities.

c) Quality policy.

d) Qualification and staff training.

e) Quality assurance.

f) Documents control.

g) Records, conservation and archiving.

h) Facilities and environment.

i) Instruments control reagents and/or appropriate consumables.

j) Analytical procedures validation.

k) Security.

1) Environmental aspects for example conveyance, consumables, waste disposal, in addition and independently to bullets $\mathrm{h}$ ) and i).

m) Research and development.

n) Procedures and analytical methods list.

o) Prescription protocols, primary samples removal, samples collection and processing.

p) Results validation.

q) Quality controls (including inter-laboratory comparison).

r) Laboratory's information system.

s) Results review.

t) Remedial actions and processing of complaints.

u) Communication and other relations with clients, health professionals, subcontracting laboratories and suppliers.

v) Internal audits.

w) Ethics

x) References ${ }^{6}$

\section{Results and discussion}

We developed a quality manual which presents the general provisions adopted and executed by the laboratory in order to guarantee the quality of its provided services in compliance with the current regulations and the NF EN ISO 15189 version August 2007 standard (Figure 2). This manual includes as well the 
laboratory structure, its different provided services and the measures implemented systematically in terms of quality assurance. It refers to the common horizontal procedures which precise operational, organizational and managerial dispositions. This manual presents the elements which, by implementing the described dispositions, demonstrate the laboratory's ability to deliver services in compliance with the regulatory requirements and clients requirements, in order to increase their satisfaction. Modifications might be carried out during the annual review or in case of important changes in the organization or the quality system. The quality manual of LAB-CAPM is a part of the laboratory's quality system documentation. It's subject to control procedures requirements notably with regard to distribution and archiving.

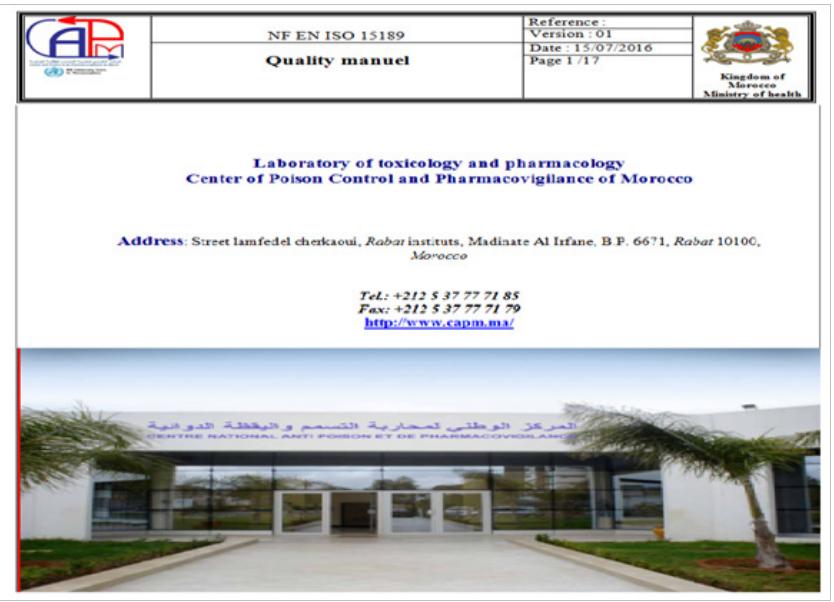

Figure 2 Quality manual cover

We produced 10 processes containing all the activities of the laboratory:
i. Laboratory general organization process
ii. Quality system follow-up process
iii. Pre-analytic process
iv. Analytics process
v. Post-analytic process
vi. Staff process
vii. Information management process
viii. Equipments maintenance process
ix. Purchasing process

x. Hygiene, security and environment process.

Many procedures (more than twenty) were elaborated; this will enable a quality increase in the whole laboratory. These procedures are documents which tackle operatory modes.

a. Internal audits procedure;

b. Non-compliance management procedure;

c. Corrective actions procedure;

d. Preventive actions procedure.

There are other procedures identified in the procedures and instructions list, which area applicable depending on the identified risk areas. The quality manual is a document which contains a set of clear and precise instructions regarding all the ongoing operations of LAB-CAPM. For the laboratory, it constitutes a memory capitalizing the individual and collective expertise. It's a reference used by the entire laboratory staff. It indicates operations processing flow, while specifying:

i. The task to do (what);

ii. The responsibility level (who);

iii. Different processing steps (when);

iv. Premises of establishment (where);

v. Execution mode (how)

The main objective of the quality manual is to express in an accessible direct language, who is doing what, where, how and for what reason? The major objectives of the quality manual are in the context of internal control, amongst others, ensure the sustainability of the laboratory.

Just like an internal control it allows the following:

a) Explain the operating mode of different procedures according to LAB-CAPM activities;

b) Safeguard the laboratory's assets by using permanent internal control procedures;

c) Promote the harmonization of tasks execution modes by formalizing it. Which excludes differences on the manner operations are treated, more often in the absence of quality manual this will depend on style, acquired personal experience and as well the nature of operations;

d) Train staff

\section{Quality manual functions}

The functions assigned to the quality manual are enumerated as follow:

i. Management tool: The quality manual defines activities execution modalities by entrusting acts and tasks to every procedures interveners.

ii. Communication tool: The manual creates conditions for different interveners to act in cooperation, following standards and common codes.

iii. Management information tool: The manual is a management information tool because it enables to directors and services managers to better analyze the information and results which are given to them in a standardized format.

\section{Structure and presentation of the quality manual}

The quality manual presentation must match content and form criterions, but also the diffusion support must be defined.

\section{Relationship of the quality manual to the validation master plan}

a. Quality Manual: The quality manual is a document that a facility writes to explain which portions of which regulations are applicable to the facility and which documents the policies, procedures, 
responsibilities and documentation that must be in place for the facility to comply with these regulatory responsibilities.

b. Validation Master Plan: The Validation Master Plan is a summary plan which communicates management's expectations and commitments to be followed for the sites validation program including the responsibilities and is therefore a key document at a site. It describes the program to be conducted to get the items in question in a validated manner. The plan lists all of the validation activities to be completed, as well as the schedule for their completion. The term validation is used to demonstrate with written evidence that the item under consideration, e.g. process does what it purports to do. Validation includes but is not limited to: equipment, computer systems, production processes, cleaning procedures, facilities, utilities as well as analytical methods.

\section{Conclusion}

The Manual is almost like all other data in the system, a reflection of two things:

a. A result: Being a descriptive of the organization as a structure committed to a process of customer satisfaction and continuous improvement of its organization.

b. A reflection: The norm attributes to it minimal characteristics (which monopolize in all that 4 lines in the chapter). ${ }^{7}$

Everybody knows that non-quality is expensive. Quality as well especially COFRAC (French Accreditation Committee) quality. ${ }^{8}$ Quality approach offer greater rigor in work methods and in the organization, a better definition of functions and responsibilities, a practices harmonization. Tracking non-compliance and dysfunction is always a priority. In this level, quality management meets risks management and prevention. It's the main purpose of quality. Likewise in every standard, writing internal quality document is inescapable and imposes to shift from an oral tradition to a written one. These necessary documents are a warranty of all actions traceability, but are not the essential. ${ }^{2}$ Preparation-implementation period for accreditation may require 2years of tedious and frustrating work. Getting accreditation is feeling of satisfaction to people who participated in the process. Post-accreditation settlement period may also take 2 years. The staff will have much better understanding after this period. Accreditation means that the serving laboratory has been successful at meeting the requirements of international accreditation standards. The patient reduces the risk of selecting an incompetent laboratory. ${ }^{9}$
The quality folder becomes a communication medium:

i. Internal: it can be used to sensitize new entrants on the organization of the laboratory

ii. External: for clients and auditors to present the general organization and give confidence to the control of the activities. ${ }^{10,11}$

\section{Acknowledgements}

None.

\section{Conflict of interest}

The authors declare there is no conflict of interest.

\section{References}

1. Daniel Pierre. L'accréditation Cofrac des laboratoires de biologie médicale dans le cadre de la loi HPST; Accreditation of the laboratories medical biology in France. Revue Francophone des Laboratoires. 2010;(149):27-28.

2. Pascal P, Beyerle F. Les référentielles qualités applicables dans les laboratoires d'analyses de biologie médicale; Quality standards for medical laboratories. Pathologie Biologie. 2006;54(6):317-324.

3. http://www.qualiblog.fr/doc

4. Standards for Medical Laboratory. Accreditation for medical laboratories (2007) CPA, Clinical Pathology Accreditation (UK) Ltd. PD-LAB Standards. 2010;2(2):1-58.

5. www.ukas.com/accreditation/cpa

6. ISO 15189:2003 Standard. Medical laboratories - particular requirements for quality and competence. Geneva: ISO.

7. ISO 15189:2007 Standard. Medical laboratories - particular requirements for quality and competence, Geneva: ISO.

8. http://nathalie.diaz.pagesperso-orange.fr/html/document/manuelqual/ indexmanuelqual.html

9. Mallet G, Dugay A, Leroy M, et al. Démarche d'accréditation Cofrac dans un laboratoire hospitalier polyvalent. Mise en place et gestion au quotidian Cofrac accreditation in a general-purpose clinical laboratory. Installation and daily management. Immuno-analyse \& Biologie Spécialisée. 2005;20(4):239-244.

10. Guzel O, Guner EI. ISO 15189 accreditation: Requirements for quality and competence of medical laboratories, experience of a laboratory I. Clin Biochem. 2009;42(4-5):274-278.

11. http://www.cala.ca/ilac_the_advantages_of_being.pdf 